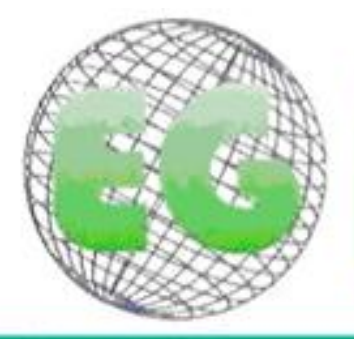

№ 33

\title{
Nivel de funcionalidad de los enfermos crónicos y su relación con la calidad de vida de los cuidadores informales, Villavicencio, Colombia, 2011
}

Chronic pacients's funcionality level and its relation with informal caregivers' quality of life, Villavicencio, Colombia, 2011

\section{*Aponte Garzón, LH **Pinzón Rocha, ML ***Galvis López, CR \\ *Grupo de investigación en Cuidado, Programa de Enfermería, Universidad de los Llanos. Colombia. E-mail: Ihaponte@hotmail.com}

\author{
Palabras clave: Enfermedad Crónica; Calidad de Vida; Cuidadores \\ Keywords: Chronic Disease; Quality of Life; Caregivers.
}

\section{RESUMEN}

Objetivo: Examinar la relación que existe entre el nivel de funcionalidad de los enfermos crónicos y el nivel de calidad de vida de sus cuidadores informales.

Método: Se desarrolló un estudio cuantitativo descriptivo observacional en cuidadores informales de enfermos con entidades crónicas. La muestra $(n=180)$ se recolectó de los cuidadores voluntarios mayores de edad que cuidaban a pacientes registrados en el Programa de Enfermedades Crónicas de seis unidades locales de salud del municipio de Villavicencio. Las mediciones de nivel de funcionalidad y calidad de vida de los cuidadores se realizaron aplicando los instrumentos PULSES versión de Gardner e instrumento de Calidad de Vida versión familiar de B. Ferrell respectivamente.

Resultados: Se encontró asociación que no alcanzó niveles de significancia entre limitación en el nivel de funcionalidad del enfermo crónico y calidad de vida del cuidador informal (OR: 1,23). Se halló asociación significativa entre deterioro de la función de miembros superiores $(\mathrm{OR} 2,25)$, de la función excretora $(\mathrm{OR} 2,31)$ y de los factores de apoyo $(\mathrm{OR}=2,13)$ y deterioro de la dimensión psicológica de la calidad de vida de los cuidadores informales.

Conclusiones: El cuidador informal se ve afectado por la condición de dependencia de la persona enferma a quien cuida y puede deteriorar su calidad de vida. La valoración y seguimiento de la calidad de vida del cuidador permitirá mejorar su autocuidado y su habilidad para cuidar al enfermo crónico.

\section{ABSTRACT}

Objective: To examine the relationship between chronic patients' functionality level and the quality of life of their informal caregivers. 
Method: An observational cross-sectional descriptive study was carried out. The sample $(n=180)$ was collected from patients caregivers volunteers registered in the Program of Chronic Illnesses of six local health units at the municipality of Villavicencio. Functionality level was measured using PULSES profile, Gardner version. Quality of life was measured by Ferrell's Quality of Life family version.

Results: An association $\mathrm{OR}=1,23$ ) between decreased functionality level among the patients and decreased quality of life among the caregivers was found, although did not reach statistical significance. A significant association was identified between upper limbs function deterioration (OR 2,25), excretory functions deterioration (OR 2,31) and of the support factors $(O R=2,13)$ and decreased in the psychological dimension of caregivers' quality of life.

Conclusions: Caregivers' quality of life is affected by deterioration on functionality level of those chronic patients who are taken care for. Evaluation and follow-up of quality of life level among caregivers' would improve self-care awareness and ability for informal care.

\section{INTRODUCCIÓN}

Las enfermedades crónicas constituyen el mayor problema de salud pública en la actualidad, son la principal causa de muerte en el mundo y se estima que para el año 2030 los años de vida perdidos por discapacidad y muerte prematura en la población de países de bajos y medianos ingresos será tres veces mayor a la suma de los años perdidos por enfermedades transmisibles, enfermedades maternas y perinatales, y alteraciones nutricionales ${ }^{(1)}$. Las enfermedades crónicas generan la mayor proporción $(63 \%)$ de carga total de enfermedad y discapacidad de América Latina y el Caribe ${ }^{(2)}$.

La atención en salud de base comunitaria, que responda a este perfil epidemiológico, debe propiciar la inclusión de los cuidadores informales de las personas con enfermedades crónicas en el sistema de salud, de manera que se aproveche y fortalezca el importante servicio que aporta para el bienestar del enfermo ${ }^{(3)}$.

Los cuidadores informales son actores principales en el cuidado de las personas con enfermedad crónica, con frecuencia ancianos con alto grado de dependencia. Los cuidadores informales son con frecuencia familiares del enfermo crónico, y suministran apoyo en el entorno domiciliario a las necesidades derivadas de su situación de salud, sin tener formación en salud y sin recibir remuneración económica $^{(4)}$. Los cuidadores aportan tiempo y esfuerzo para ofrecer a la persona con enfermedad crónica los cuidados básicos en el domicilio, y frecuentemente, el cuidado al enfermo transforma la vida del cuidador al punto en que solo se ocupa del enfermo y a menudo de cuidar a otros miembros de la familia sin atender sus necesidades de cuidado, con lo que se puede presentar un cambio en el nivel calidad de vida del cuidador $^{(5)}$.

El propósito de esta investigación fue la de explorar la relación entre el nivel de funcionalidad o dependencia de la persona con enfermedad crónica que recibe el cuidado y la calidad de vida del cuidador informal que brinda el cuidado.

\section{MATERIAL Y MÉTODO}

La investigación tuvo un diseño cuantitativo, observacional, descriptivo, transversal. El universo estuvo compuesto por los cuidadores de personas con enfermedad crónica que residen en el municipio de Villavicencio. La muestra $(n=180)$ estuvo conformada por cuidadores de personas con enfermedades crónicas que cumplieron con los siguientes criterios de inclusión: Ser mayor de 18 años, participante voluntario 
en el estudio y ser cuidador informal de una persona en situación de enfermedad crónica, inscrito durante el período de estudio en el Programa de Promoción y Prevención de seis instituciones de salud del nivel local pertenecientes a la Empresa Social del Estado (ESE) del municipio de Villavicencio, Colombia.

Todos los cuidadores informales expresaron su voluntad de participar en el estudio firmando el respectivo consentimiento informado de conformidad con lo establecido en la Resolución 08430 de 1993 del Ministerio de Salud ${ }^{(6)}$. Esta investigación se clasificó como investigación sin riesgo debido a la aplicación de un cuestionario en la que no se realiza una intervención o modificación intencionada de variables biopsicosociales y no se identifica al participante.

La selección de los participantes se hizo por método no probabilístico, de tipo intencional o por conveniencia. La unidad de muestreo está constituida por cada cuidador informal participante en el estudio. La unidad de análisis es la misma unidad de muestreo.

Para la recolección de información se utilizaron dos instrumentos: El Instrumento de Medición de Calidad de Vida de Ferrell ${ }^{(7)}$ y la escala de funcionalidad PULSES ${ }^{(8)}$. El instrumento de Calidad de Vida tiene 37 ítems en escala de Likert que describen las dimensiones físicas, psicológicas, espirituales y sociales de la calidad de vida. Este instrumento tiene un grado de validez y confiabilidad de 0.89 , la consistencia presentó un alfa de 0.69 en los cuatro dominios. Se contó con la autorización del autor para el uso del instrumento en esta investigación. Los 37 ítems tienen puntajes de 1 a 4, de manera que los resultados posibles están entre un mínimo de 37 y un máximo de 148 puntos. Un nivel de deficiencia en calidad de vida se estableció en puntajes menores o iguales a 74, regular en puntajes entre 75 a 110, y buena calidad de vida con puntajes iguales o mayores a 111.

La escala PULSES es un instrumento de valoración de funcionalidad, adaptado por Granger et al ${ }^{(9)}$, usado ampliamente en el seguimiento de pacientes en post-evento cerebro vascular y usado también en la valoración de la funcionalidad de las personas con enfermedad crónica. El instrumento tiene una validez y confiabilidad similar a la del Índice de Barthel (validez 0,73; alpha de Cronbach 0,90) siendo capaz de diferenciar los distintos grados de discapacidad y describir la funcionalidad conservada $^{(10)}$, particularmente en la versión propuesta por Shah et al ${ }^{(11)}$. La escala PULSES, en forma similar a la versión de IB de Shah está organizada en puntajes diferentes para cada categoría manteniendo una escala corta, limitando la pseudoprecisión que puede tener lugar cuando se usan escalas más amplias como la de Barthel original de 0 a 100. De otro lado, se debe advertir que en general la valoración de la funcionalidad por parte de personas próximas o cuidadores informales presenta una tendencia a obtener valores ligeramente menores o los de la real capacidad funcional ${ }^{(12)}$.

EI PULSES consiste en una escala de medición nominal de seis parámetros con valores posibles de 1 a 4 para cada parámetro en donde 1 representa la ausencia de limitación en la funcionalidad y 4 la mayor limitación en la funcionalidad. Cada parámetro mide un aspecto particular de funcionalidad así: el parámetro $P$ (de pshysical condition) valora el grado de estabilidad de la patología examinada por la cantidad de supervisión por parte de los profesionales de salud que el enfermo requiere de manera regular, el parámetro $U$ (por Upper limb function) valora la función de las miembros superiores y refleja la necesidad de asistencia para las acciones de 
autocuidado, tales como comer, beber, vestirse, asearse, etc. El parámetro L (Lower limb function) valora la funcionalidad de los miembros inferiores y está relacionada con la movilidad y el desplazamiento, el parámetro $S$ (Sensory componentes) evalúa la función sensorial, vista, oído y la capacidad para la comunicación, el parámetro E (por Excretory functions) valora control de esfínteres y señala la necesidad de asistencia en estas funciones. Por último, el Parámetro $S$ (por Support) examina los factores de apoyo de tipo psicológico, social, familiar, etc., con que cuenta el enfermo, por lo que está indicando la necesidad de prestar asistencia relacionada con dificultades de orden social y psicológico. El instrumento establece que un puntaje de 6 señala la presencia de total independencia, a un aumento del puntaje corresponde un aumento de la dependencia del enfermo, un puntaje de 24 indica dependencia total. La interpretación por rangos establece que un puntaje final entre 6 a 8 indica nivel funcional conservado, entre 9 a 11 indica compromiso leve de la funcionalidad, puntajes iguales o mayores a 12 identifican casos de severo compromiso de la funcionalidad con alto grado de dependencia.

Los entrevistadores, estudiantes de último año del Programa de Enfermería de la Universidad de los Llanos, recibieron formación específica sobre el uso de ambos instrumentos y sobre las técnicas de la entrevista en cuatro talleres diferentes, cada uno con una duración aproximada de 2 horas, en donde se desarrollaron técnicas expositivas y de simulación práctica. Los instrumentos se aplicaron a los participantes por medio de entrevista guiada de manera que los términos fueran comprendidos por los cuidadores para mejorar así la calidad de la respuesta. La información recolectada se analizó con el software Excel 2007 y SPSS 17. Para el análisis estadístico, se aplicaron medidas de estadística descriptiva, se realizó estimación de asociación usando la razón de posibilidades (OR), con aplicación de pruebas de Chi cuadrado con un valor de significancia de $p=0,05$.

Como beneficio por participación y una vez finalizada la recolección, se ofreció a los cuidadores informales información actualizada sobre el cuidado a personas con enfermedades crónicas.

\section{RESULTADOS}

La Tabla 1 muestra la distribución de la muestra por variables edad y género. Obsérvese que la gran mayoría de los cuidadores son de género femenino $(78.89 \%)$ y oscilan entre los 31 y 70 años de edad.

Tabla 1. Distribución de los cuidadores informales por edad y género. Villavicencio, Colombia. 2011

\begin{tabular}{lrrrrrr}
\hline EDAD & FEMENINO & \multicolumn{1}{c}{$\%$} & MASCULINO & $\%$ & TOTAL & \multicolumn{1}{c}{$\%$} \\
\hline $18-30$ & 28 & 19,72 & 5 & 13,16 & 33 & 18,33 \\
$31-50$ & 69 & 48,59 & 12 & 31,58 & 81 & 45,00 \\
$51-70$ & 39 & 27,46 & 14 & 36,84 & 53 & 29,44 \\
$71 \mathrm{y}+$ & 6 & 4,23 & 7 & 18,42 & 13 & 7,22 \\
\hline & 142 & 78,89 & 38 & 21,11 & 180 & 100,00 \\
\hline
\end{tabular}

Los cuidadores viven en estratos socioeconómicos de nivel $1(25 \%), 2$ (47\%) y 3 $(28 \%)$. Lo anterior señala la existencia de restricciones importantes en acceso a bienes y servicios que pudieran aliviar la carga del cuidado en los cuidadores informales. La gran mayoría de los cuidadores informales son casados o viven en 
unión libre con sus parejas $(63,89 \%)$, un $21,11 \%$ es soltero, una pequeña proporción es separado o viudo (15\%).

Los datos reportados apoyan la afirmación de que los cuidadores son principalmente mujeres, mayores de 31 años, casadas o en unión libre. Las cuidadoras informales en su mayoría cuidan de uno o ambos padres $(47,78 \%)$ o de sus parejas $(25,56 \%)$.

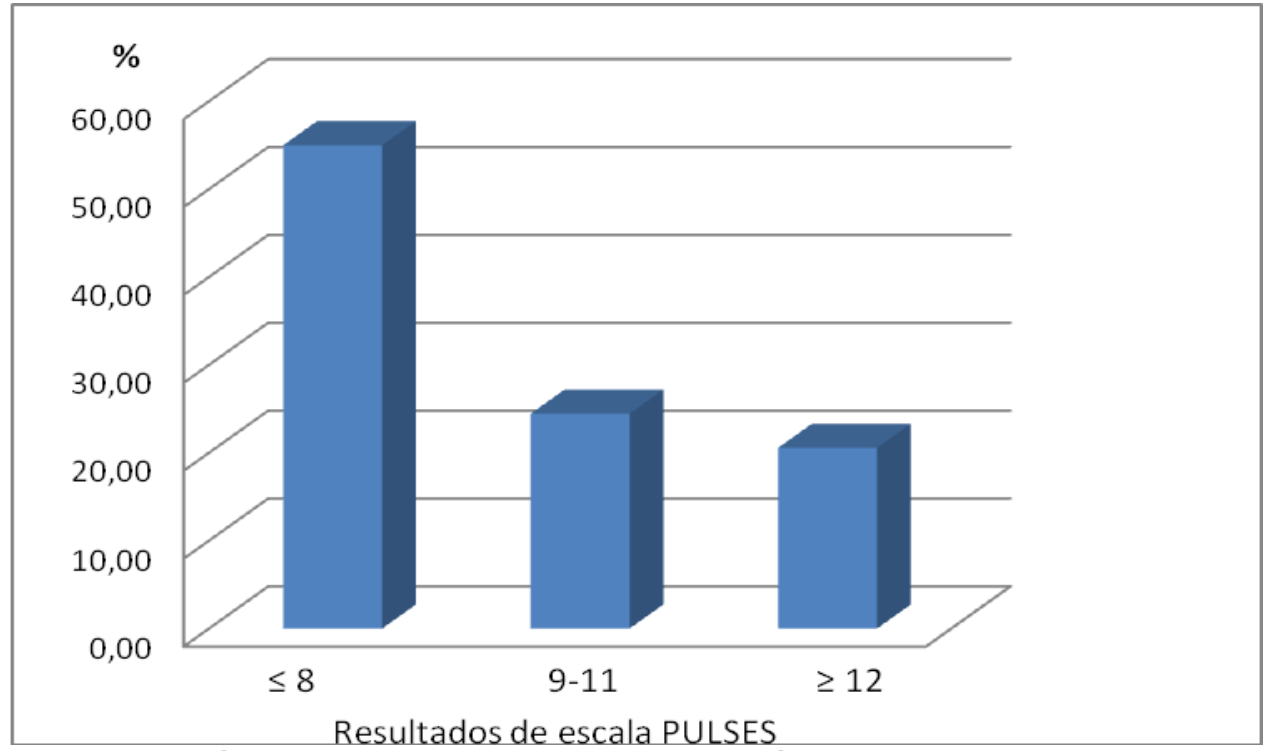

Figura 1. Distribución porcentual de los enfermos crónicos que reciben el cuidado según resultados globales de la escala PULSES. Villavicencio, Colombia. 2011

Los valores globales de PULSES en los enfermos crónicos reportan una media de 9,12 (DS $\pm 2,95$ ) con unos valores mínimo y máximo de 6 y 22 respectivamente. La Figura 2 reporta la distribución de la muestra con respecto a la valoración global de funcionalidad. La distribución entre los enfermos con independencia o con limitaciones que no afectan de manera significativa la funcionalidad es similar al grupo de enfermos con limitaciones leves y severas. De otro lado, frente al valor de 6 que representa total independencia se encuentra que tan solo el $13,89 \%$ son clasificados como personas con total independencia y la gran mayoría, correspondiente al $86,11 \%$ de los enfermos, son clasificados como personas con algún grado de dependencia.

Tabla 2. Distribución de resultados de la escala PULSES de nivel de funcionalidad de enfermos crónicos por parámetro de medición, Villavicencio, Colombia. 2011

\begin{tabular}{|c|c|c|c|c|c|c|}
\hline PARÁMETRO & $\begin{array}{l}\text { CONDICIÓN } \\
\text { FÍSICA (\%) }\end{array}$ & $\begin{array}{c}\text { FUNCIÓN } \\
\text { MIEMBROS } \\
\text { SUPERIORES } \\
(\%)\end{array}$ & $\begin{array}{c}\text { FUNCIÓN } \\
\text { MIEMBROS } \\
\text { INFERIORES } \\
(\%)\end{array}$ & $\begin{array}{c}\text { COMPONENTES } \\
\text { SENSORIALES } \\
(\%)\end{array}$ & $\begin{array}{c}\text { FUNCIÓN } \\
\text { EXCRETORA } \\
(\%)\end{array}$ & $\begin{array}{c}\text { FACTORES } \\
\text { DE APOYO } \\
(\%)\end{array}$ \\
\hline \multicolumn{7}{|l|}{ PUNTAJE } \\
\hline 1 & $99(55)$ & $130(72,2)$ & $112(62,2)$ & $52(28,9)$ & $135(75)$ & $93(51,7)$ \\
\hline 2 & $68(37,8)$ & $37(20,6)$ & $43(23,9)$ & $114(63,3)$ & $31(17,2)$ & $76(42,2)$ \\
\hline 3 & $12(6,7)$ & $11(6,1)$ & $20(11,1)$ & $13(7,2)$ & $11(6,1)$ & $11(6,1)$ \\
\hline 4 & $1(0,6)$ & $2(1,1)$ & $5(2,8)$ & $1(0,6)$ & $3(1,7)$ & $0(0)$ \\
\hline
\end{tabular}

La Tabla 2 muestra los resultados de la escala PULSES por parámetro evaluado que señala el origen y tipo de dependencia y sugiere el tipo de apoyo que es suministrado por el cuidador en forma cotidiana. En los casos de deterioro leve del nivel de funcionalidad se observa compromiso del parámetro de componentes sensoriales evidenciando una limitación del enfermo para la comunicación con su entorno. Igualmente, los datos reportan la existencia de compromiso en los factores de apoyo disponibles para el desarrollo de sus actividades y roles. En casos de puntajes que 
muestras limitaciones más marcadas estas se relacionan con deterioro de la función de miembros inferiores, de movilidad y de capacidad para deambular, lo que coloca al enfermo en situación de alta dependencia y exige del cuidador informal una atención continua. En los estados de mayor compromiso de la funcionalidad o máxima severidad se combinan el deterioro de la movilidad, del control de la función excretora y de la movilidad de miembros superiores con limitación severa de las capacidades de autocuidado del enfermo, que evidentemente deben ser suplidas por el cuidador.

Los resultados de Calidad de Vida en los tres niveles, indican que más de la mitad $(52,78 \%)$ de los cuidadores reportan un nivel de calidad de vida regular o deficiente. El restante $47.22 \%$ reportan buena calidad de vida.

Sin embargo, al observar la relación que existe entre nivel de funcionalidad del enfermo que recibe el cuidado y el nivel de la calidad de vida del cuidador (Tabla 3) se encuentra asociación entre nivel de funcionalidad del enfermo y calidad de vida del cuidador, en donde la exposición corresponde a valores de PULSES mayores a 8 indicando niveles de afección de la funcionalidad de leves a severos y el evento bajo estudio corresponde a calidad de vida deficiente, es decir, resultados de calidad de vida global iguales o menores a 74. La estimación de riesgo (OR) de deficiente calidad de vida del cuidador en presencia de algún déficit en el nivel de funcionalidad del enfermo crónico es de 1,23 (OR), sin embargo, esta asociación no alcanza niveles de significancia. (Valor $p=0,49$ ).

Tabla 3. Asociación entre Valores Globales de Nivel de Funcionalidad del Enfermo Crónico y Calidad de Vida de los Cuidadores Informales, Villavicencio, Colombia, 2011.

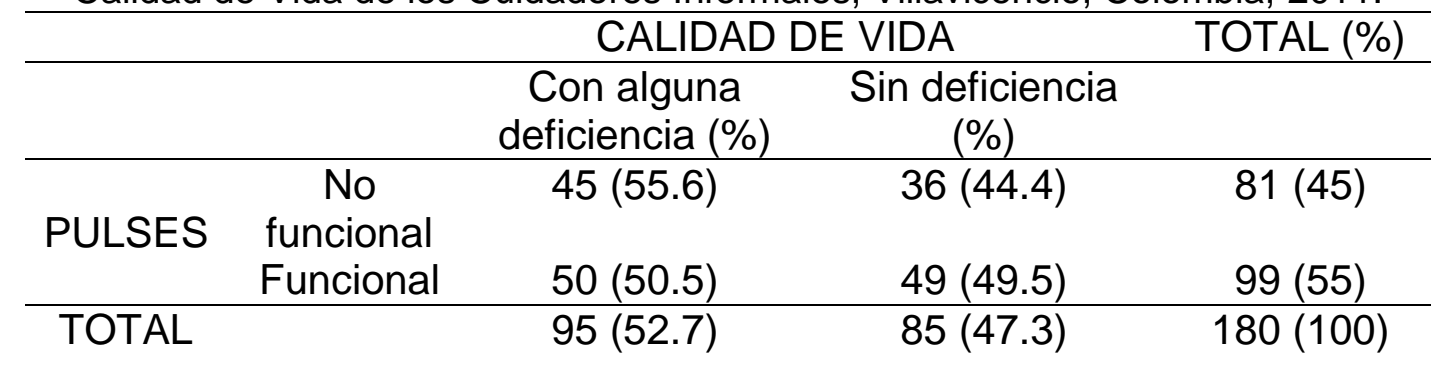

Al examinar la relación entre los parámetros de nivel de funcionalidad y los ítems de calidad de vida en cada dimensión se encontró compromiso de las dimensiones de Bienestar Psicológico, Bienestar Social y Espiritual. El Bienestar Psicológico fue el más afectado y los resultados del análisis de los elementos que integran esta dimensión en relación con los parámetros de funcionalidad del enfermo crónico se presentan en la Tabla 4. Esta tabla presenta los resultados de estimación de riesgo de deterioro de la dimensión psicológica en el cuidador informal cuando cuida a una persona con enfermedad crónica. Los aspectos afectados dentro de la dimensión psicológica fueron la presencia de aflicción y de angustia a causa del diagnóstico y de los tratamientos del enfermo. 
Tabla 4. Relación entre los criterios de bienestar psicológico de los cuidadores informales y los parámetros de funcionalidad de los enfermos crónicos, Villavicencio, Colombia, 2011.

\begin{tabular}{ccccc} 
Resultado PULSES & \multicolumn{4}{c}{ Resultado Calidad de Vida } \\
\hline Parámetro valorado & $\begin{array}{c}\text { Disminución en el bienestar psicológico de } \\
\text { los cuidadores informales* }\end{array}$ \\
\hline $\begin{array}{c}\text { Deterioro de la función de } \\
\text { miembros superiores }\end{array}$ & $X^{2}$ & Valor p & OR & IC \\
$\begin{array}{c}\text { Deterioro de la función } \\
\text { excretora }\end{array}$ & 5.789 & 0.016 & 2.25 & $1.15-4.38$ \\
$\begin{array}{c}\text { Deterioro en los factores } \\
\text { de apoyo }\end{array}$ & 6.14 & 0.015 & 2.31 & $1.16-4.60$ \\
& 0.013 & 2.13 & $1.16-3.90$ \\
\hline
\end{tabular}

* Relacionado con presencia de aflicción y angustia causados por los tratamientos al enfermo.

\section{DISCUSIÓN}

La relación entre la funcionalidad del enfermo crónico y la calidad de vida del cuidador es un asunto relevante en el campo de enfermería considerando la alta relevancia del rol que el cuidador tiene frente al cuidado del paciente crónico. El cuidador informal suministra un apoyo muy importante en el bienestar del enfermo crónico en aspectos relacionados con la administración de medicamentos, alimentación, movilidad y aseo, además del apoyo afectivo de alta significancia en las personas con enfermedades de larga duración quienes con frecuencia sufren además aislamiento y pérdida de sus redes sociales.

Así, un estudio comparativo ${ }^{(13)}$ de cuidadores informales y no cuidadores informales mostró la mayor presencia de enfermedad manifestado por visitas médicas y pérdida de peso en el último año en las personas que cuidan de enfermos crónicos, siendo las mujeres cuidadoras quienes mostraron un riesgo aumentado (OR 1,5-2) de presentar síntomas diversos como cefaleas, mareos, inestabilidad emocional y alteración en el sueño cuando se comparaban con mujeres no cuidadoras informales. De igual manera, un estudio ${ }^{(14)}$ desarrollado en cuidadores de personas ancianas señaló que cerca del $46 \%$ de los cuidadores tenían sobrecarga relacionada con el rol de cuidador y reportó la existencia de una asociación significativa entre el nivel de dependencia del enfermo y la disminución de la calidad de vida de los cuidadores.

Adicionalmente, una investigación ${ }^{(15)}$ realizada en cuidadores de cónyuge adulto mayor con pérdida progresiva de la funcionalidad, señala la progresión de cuadros de tristeza crónica en los cuidadores. La respuesta inicial ante la pérdida de la salud de la pareja genera en el cuidador una conducta de duelo que no siempre es posible experimentar de manera reflexiva ya que se deben afrontar las necesidades de organización y función que el enfermo continuamente requiere, en este escenario, el progreso de la enfermedad manifestada con la pérdida de la funcionalidad, ampliación de los esquemas terapéuticos o limitaciones en el tipo y frecuencia de actividades que antes desarrollaban, imponen en el cuidador una situación crónica de duelo que se reproduce con el curso de la enfermedad crónica del enfermo. En forma similar, López et al. ${ }^{(16)}$ encontraron mayor prevalencia de ansiedad, depresión y percepción de mala salud en los cuidadores (OR 2,02; $\mathrm{Cl} 1,27-3,21$ ) comparados con personas que no desempeñaban ese rol. 
Los hallazgos de este estudio sobre la relación entre el nivel de funcionalidad de los enfermos crónicos y la condición del cuidador son concordantes con otros estudios que señalan el impacto negativo que sobre el cuidador informal tiene el cuidado de las personas con detrimento de la funcionalidad.

Estos resultados son similares a los que el presente estudio muestra, en el que se señala que la presencia de asociación entre disminución de funcionalidad del enfermo crónico y disminución de calidad de vida percibida por el cuidador informal. De igual manera, se evidencia el impacto que el cuidado del enfermo crónico con diversos niveles de pérdida de funcionalidad tiene sobre el bienestar psicológico del cuidador, de manera particular, en lo relacionado con los tratamientos dados al enfermo, que con frecuencia se deben ajustar y hacer cada vez más complejos, en la medida en la que la enfermedad crónica progresa.

En este aspecto, un estudio ${ }^{(17)}$ realizado sobre predictores de calidad de vida en cuidadores de personas con enfermedades neurológicas que generan diversos grados de dependencia reportó que el ánimo y el estrés psicológico fueron identificados como predictores significativos de calidad de vida de los cuidadores de personas con los tipos de alteraciones neurológicas estudiadas, similares hallazgos se reportaron en cuidadores de pacientes que han sufrido eventos cerebrovasculares $^{(18)}$. De esto modo, se encuentra consistencia entre los hallazgos del presente estudio y lo reportado en la literatura sobre la presencia de cambios en el bienestar psicológico del cuidador de personas con enfermedad crónica, de manera particular, cambios en el ánimo por presencia de ansiedad y aflicción asociado a los cambios en los tratamientos derivados del progreso de la enfermedad.

La problemática asociada con la deficiente calidad de vida y problemas psicológicos de los cuidadores informales de personas con enfermedad crónica es de alta relevancia para el sistema de salud y debe ser abordado con medidas preventivas en el primer nivel de atención. En la actualidad, se ha reconocido la importancia del cuidador informal en el mantenimiento del bienestar del enfermo crónico y algunos sistemas de salud han desarrollado estrategias para integrar al cuidador al sistema facilitando la obtención ágil de la información necesaria para dar un cuidado seguro y ampliando el acceso a los servicios que tanto el enfermo como el cuidador requieren, facilitando de esta manera que el personal de salud reconozca y apoye la labor del cuidador ${ }^{(19,20)}$.

\section{CONCLUSIÓN}

Basados en los resultados ya discutidos se recomienda que se continúen y fortalezcan el desarrollo de estrategias de programas que involucren a los cuidadores en el equipo de cuidado, eliminando barreras entre los cuidadores y el sistema de salud, de modo que pueda ofrecer el cuidado en el domicilio disponiendo de la información necesaria relacionada con los cambios en el tratamiento, avances de la enfermedad y expectativas de progreso del cuadro clínico. Se recomienda también, la vinculación de los cuidadores a programas organizados de capacitación ${ }^{(21)}$ y a redes de apoyo de forma que pueda fortalecer su habilidad de cuidado y contrarrestar el estrés psicológico y aislamiento social que presentan los cuidadores de pacientes crónicos. 


\section{Agradecimientos}

Las autoras agradecen a la Universidad de los Llanos por el soporte financiero para el desarrollo de esta investigación a través de la Dirección General de Investigaciones, Universidad de los Llanos. Agradecen también a los estudiantes del Programa de Enfermería por su participación en el desarrollo de este estudio como estudiantes en opción de grado.

\section{REFERENCIAS BIBLIOGRÁFICAS}

1. Organización Mundial de la Salud. Burden: mortality, morbidity and risk factors. Capítulo 1 En: Global status report on communicable diseases 2010. 2011 World Health Organization. [consultado 2011 Nov. 23] 2011;9-31. Disponible en: http://whqlibdoc.who.int/publications/2011/9789240686458 eng.pdf

2. Organización Panamericana de la Salud. Vigilancia de enfermedades crónicas en las Américas. [aprox. 8 pantallas]. 2011 Organización Panamericana de la Salud. [consultado 2011 Nov. 23]. Disponible en: http://new.paho.org/hq/index.php?option=com content\&task=view\&id=1512\&ltemid=1 663

3. Hoskins I, Kalache A, Mende S. Hacia una atención primaria de salud adaptada a las personas de edad. Rev Panam Salud Publica [consultado 2012 Jun. 20] 2005;17(5/6):444-451. Disponible en: http://www.scielosp.org/pdf/rpsp/v17n56/26282.pdf

4. Giraldo CM, Franco GM, Correo LS, Salazar MO, Tamayo AM. Cuidadores familiares de ancianos: quienes son y cómo asumen este rol. Revista de la Facultad Nacional de Salud Pública. [consultado 2012 Jun. 20]2005;23(2):7-15. Disponible en: http://redalyc.uaemex.mx/redalyc/pdf/120/12011106002.pdf

5. Ocampo JM, Herrera J, Torres P, Rodríguez JA, Loboa L, García CA. Sobrecarga asociada con el cuidado de ancianos dependientes, Colombia médica [en línea] 2007, [consultado 2012 Ene 20]; 38(1). Disponible en: http://redalyc.uaemex.mx/redalyc/pdf/283/28338107.pdf

6. República de Colombia. Ministerio de Salud. Resolución 008430 de 1993. [consultado 2012 Jul. 8] Disponible en: http://www.dib.unal.edu.co/promocion/etica_res_8430_1993.pdf

7. Ferrell B, Grant M. Quality of life family version. City of Hope. National Medical Center and Beckman Research Institute. Disponible en: http://prc.coh.org/pdf/Quality\%20of\%20Life\%20Family\%20Version.pdf

8. Granger C, Greer D, Liset E, Coulombe J, O'Brien E. Measurements of outcomes of care for stroke patients. Stroke [serie en Internet]. 1975 Enero [citado 2011 Nov 22 ];6:[aprox.9 p.]. Disponible en: http://stroke.ahajournals.org/cgi/reprint/6/1/34

9. Granger CV, Greer DS, Liset E, Coulombe J, O'Brien E. Measurements of outcomes of care for stroke patients. Stroke 1975;6:34-41. Disponible en: http://stroke.ahajournals.org/content/6/1/34.full.pdf+html

10. Granger CVC, Albrecht GLG, Hamilton BBB. Outcome of comprehensive medical rehabilitation: measurement by PULSES profile and the Barthel Index. Arch Phys Med Rehabil 1979;60(4):145-154. Disponible en: http://search.proquest.com/health/docview/74707087/138AA9984003C2187F6/2?acco untid $=48773$

11. Shah S, vanclay F, Cooper B. Improving the sensitivity of the Barthel Index for stroke rehabilitation. J Clin Epidemiol. 1989;42(8):703-9. Disponible en: http://www.ncbi.nlm.nih.gov/pubmed/2760661 
12. Cid-Ruzafa J, Damián-Moreno,J.J. Valoración de la discapacidad física: el Indice de Barthel. Revista española de salud pública 1997;71(2):127-137. Disponible en: http://scielo.isciii.es/scielo.php?script=sci_arttext\&pid=S1135-57271997000200004 13. Ho SC, Chan A, Woo J, Chong P, Sham A. Impact of Caregiving on Health and Quality of Life: A Comparative Population-Based Study of Caregivers for Elderly Persons and Noncaregivers. The Journals of Gerontology [consultado 2012 Jul. 8] 2009;64A(8):873-879.

Disponible

en: http://search.proquest.com/health/docview/208620023/abstract/138A158F644452F2C $4 \mathrm{~F} / 1$ ? accountid $=48773$

14. Ocampo JM, Herrera JA, Torres P, Rodríguez JA, Loboa L, García CA. Sobrecarga asociada con el cuidado de ancianos dependientes. Colombia médica. [consultado 2012 Jul. 8] 2007;38(1):40-46 Disponible en: http://redalyc.uaemex.mx/redalyc/pdf/283/28338107.pdf

15. Rossheim BN, McAdams,Charles R., I.,II. Addressing the Chronic Sorrow of LongTerm Spousal Caregivers: A Primer for Counselors. Journal of Counseling and Development : JCD 2010;88(4):477-482. Disponible en: Rossheim BN, McAdams,Charles R., I.,Il. Addressing the Chronic Sorrow of Long-Term Spousal Caregivers: A Primer for Counselors. Journal of Counseling and Development : JCD 2010;88(4):477-482.

16. López Gil Mำ Jesús, Orueta Sánchez Ramón, Gómez-Caro Samuel, Sánchez Oropesa Arancha, Carmona de la Morena Javier, Alonso Moreno Francisco Javier. EI rol de Cuidador de personas dependientes y sus repercusiones sobre su Calidad de Vida y su Salud. Rev Clin Med Fam [revista en la Internet]. [consultado 2012 Ago. 25] 2009 Jun; 2(7): 332-339. Disponible en: http://scielo.isciii.es/pdf/albacete/v2n7/original3.pdf

17. O'connor E,J., Mccabe MP. Predictors of quality of life in carers for people with a progressive neurological illness: a longitudinal study. Quality of Life Research. [consultado 2012 Jul. 15] 2011;20(5):703-11. Disponible en: http://search.proquest.com/health/docview/868638392/abstract/138A1C8C77811A843 E8/14? accountid $=48773$

18. Carod-artal F, Ferreira Coral L, Trizotto DS, Menezes Moreira C. Burden and Perceived Health Status Among Caregivers of Stroke Patients. Cerebrovascular Diseases [consultado 2012 Jul. 15] 2009;28(5):472-80. Disponible en: http://search.proquest.com/health/docview/221225205/abstract/138A1C8C77811A843 E8/18?accountid $=48773$

19. Mitnick S, Leffler C, Hood VL. Family Caregivers, Patients and Physicians: Ethical Guidance to Optimize Relationships. Journal of General Internal Medicine [consultado 2012 Jul. 15] 2010;25(3):255-60. Disponible en: http://search.proquest.com/health/docview/871259893/abstract/138A1C8C77811A843 E8/9?accountid $=48773$

20. Goldberg A, Rickler KS, M.S.W. The Role of Family Caregivers for People with Chronic Illness. Medicine and Health Rhode Island [consultado 2012 Jul. 15] 2011;94(2):41-2. Disponible en: http://search.proquest.com/health/docview/862377947/citation/138A1C8C77811A843 E8/10?accountid $=48773$

21. Sapag J, Lange I, Campos S, Piette J. Estrategias innovadoras para el cuidado y el autocuidado de personas con enfermedades crónicas en América Latina. Rev. Panam. Salud Pública. [consultado 2012 Ago. 25] 2010;27(1):1-9. Disponible en: http://www.scielosp.org/pdf/rpsp/v27n1/01.pdf 
๑ COPYRIGHT Servicio de Publicaciones - Universidad de Murcia 